\title{
Prominant squamous metaplasia with extensive myxoid degeneration and associated ischaemic infarction in fibroadenoma of breast: A diagnostic dilemma
}

\author{
Poonam Prasad Bhadani ${ }^{1, *}$, Iffat Jamal ${ }^{2}$, Nishir Ojha ${ }^{3}$, Rakesh Kumar Gupta ${ }^{4}$ \\ ${ }^{\mathbf{1}}$ Additional Professor and Head, ${ }^{\mathbf{2 , 3}}$ Senior Resident, ${ }^{\mathbf{4}}$ Assistant Professor, ${ }^{\mathbf{1 - 4}}$ Dept. of Pathology, All India Institute of Medical \\ Sciences, Patna, Bihar, India \\ *Corresponding Author: \\ Email: bhadanipunam@gmail.com
}

\begin{abstract}
Fibroadenoma is the most commonly diagnosed benign tumour in adolescents and young women. Squamous metaplasia and prominent myxoid degeneation with associated ischaemia infarction is not a common finding without history of trauma in fibroadenoma. We report a 16 year old unmarried female which posed a diagnostic dilemma due to presence of unusual histopathological findings, which is sparsly reported in English literature.
\end{abstract}

Keywords: Breast, Fibroadenoma, Infarction, Squamous metaplasia, Myxoid degeneration.

\section{Introduction}

Fibroadenomas are the most common benign neoplasms of the breast usually seen in adolescents and young women. Infarction in benign breast lesions is rare and may occur in various conditions, including fibroadenomas and intraductal papilloma. ${ }^{1}$ Infarction generally occurs in young women during pregnancy or lactation, but may occur at any age following fineneedle aspiration cytology (FNAC). Spontaneous infarction in fibroadenoma occurs very rarely with associated squamous metaplasia and prominent myxoid degenerative changes. Very few cases have been reported so far in literature. ${ }^{2}$ Here we describe a case reportof spontaneous infarction in fibroadenoma with associated metaplastic change in a young adolescent patient unrelated to any known risk factor.

\section{Case Report}

A 16 year old female patient presented with a breast lump in left upper quadrant of right breast for one year which had become painful since last three months. On clinical examination the lump was 4 x 3 $\mathrm{cm}$, well defined, firm, mobile and tender. No history of fever, trauma or prior fine needle aspiration procedure. Nipple discharge, retraction or axillary lymphadenopathy was not seen with normal contralateral breast ultrasonography revealed a well defined lesion of size $4.5 \times 4 \mathrm{~cm}$ and categorized as BIRADS II.

Excision biopsy of the lump was done and sent for histopathological examination. Grossly, lump was well encapsulated measuring $4 \times 3.2 \times 2.2 \mathrm{~cm}$. Cut section was solid, greyish white, glistening with foci of hameorrahge at periphery and few tiny cystic areas. (Fig. 1) Microscopic examination revealed a well encapsulated mass having viable compressed ducts at the periphery, fair number of proliferated blood vessels were noted with large areas of ischemic necrosis. Squamous metaplasia of ductal epithelium was seen frequently with an areas of hyalinization and myxoid changes. (Fig. 2-4) No inflammatory cells or cellular atypia was seen. A strong possibility of sialometaplasia of salivary gland like tumor was considered and did extensive grossing, which showed similar microscopic features in representative multiple sections. A histotopathological diagnosis of benign proliferative breast disease favouring fibroadenoma with subtotal infarction along with extensive squamous metaplasia was made with advise of close follow up. No recurrence or any other complains is noted after 6 month of follow up.

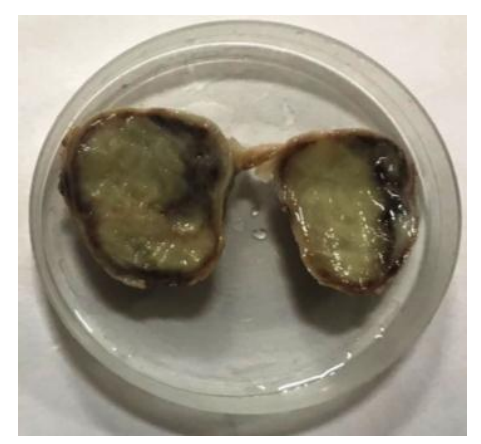

Fig. 1: Cut section shows a well encapsulated solid glistening, greyish white mass having tiny cystic areas with areas of hameorrhage and necrosis at the periphery

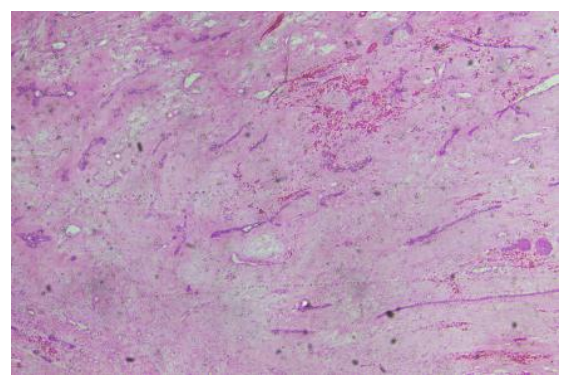

Fig. 2: Microphotograph showing compressed viable ducts at the periphery with haemorrhagic foci with myxoid degeneration 


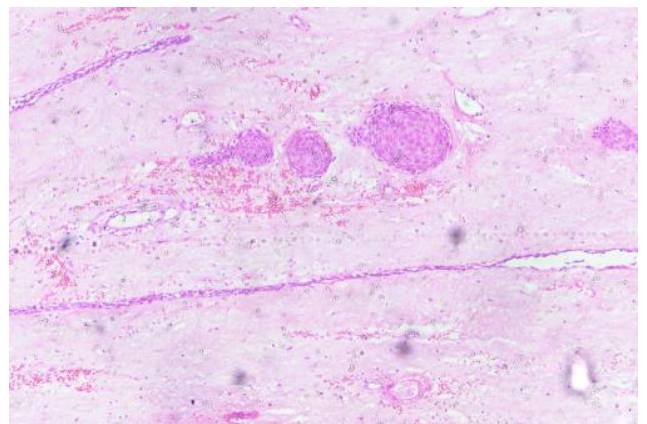

Fig. 3: Microphotograph showing squamous metaplasia of ducts

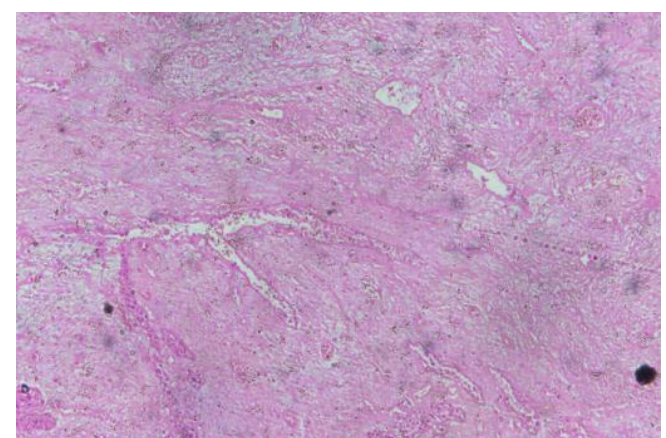

Fig. 4: Microphotograph showing proliferated blood vessels with an areas of ischaemic necrosis

\section{Discussion}

Spontaneous infarction within fibroadenoma is a rare complication of benign breast tumour. It constitutes the most characteristic example of necrosis of the breast tissue.

Delaure and Redon in 1949 first described spontaneous infarction in fibroadenoma in young females. ${ }^{1,2}$ Infarction within a fibroadenoma of the breast is an uncommon entity, noted in $0.5 \%$ to $1.5 \%$ of all cases including those seen in pregnant and lactating women or after a recent FNAC. ${ }^{3}$ Rarely, it can be seen in young patients without any associated risk factors, as illustrated. The cause and mechanism of infarction are largely unknown. One of the possible explanations is that infarction represents a spectrum of regressive changes that also may include calcification and hyalinization, both of which are much more commonly seen in fibroadenomas. ${ }^{4}$ Areas of squamous metaplasia resembling so called necrotizing sialometaplasia of salivary glands is also a commonly seen. This phenomenon has been described in infarcted fibroadenoma in a close proximity to the area of infarction. ${ }^{5}$ Presence of extensive ischemic necrosis, very few viable ducts and large areas of squamous metaplastic changes pose a diagnostic difficulty. The differential diagnosis includes underlying breast malignancy, infarcted intraductal papilloma due to presence of large areas of ischemic necrosis and Pleomorphic adenoma due to presence of extensive squmaous metaplasia and stromal hyalinization and myxoid changes. ${ }^{67}$ To conclude the findings, spontaneous infarction is a rare event in fibroadenomas and may not be associated with any known risk factor. It is an uncommon complication within fibroadenoma and poses diagnostic dilemma and hence clinicians, radiologists and pathologists must be aware of this entity to avoid misinterpreting as malignancy or sialometaplasia like feature as seen in salivary glands.

\section{References}

1. Meerkotter D, Andronikou S: Unusual presentation and inconclusive biopsy render fibroadenoma in two young females a diagnostic dilemma: case report. SA Journal of Radiology 2009, 13:62-65.

2. Onuigbo W: Breast fibroadenoma in teenage females. Turk J Pediatr 2003, 45:326-328.

3. Al-Atrooshi SA: Fibroepithelial tumors of female breast: a review of 250 cases of fibroadenomas and phylloides tumors. The Iraqi Postgraduate Medical Journal 2012, 11:140-145.

4. Verslegers I, Tjalma W, Van Goethem M, Colpaert C, Biltjes I, De Schepper AM, Parizel PM: Massive infarction of a recurrent phyllodes tumor of the breast: MRI-findings. JBR-BTR 2004, 87:21-22.

5. Deshpande KM, Deshpande AH, Raut WK, Lele VR, Bobhate SK. Diagnostic difficulties in spontaneous infarction of a fibroadenoma in an adolescent: Case report. Diagn Cytopathol 2002;26:26-8.

6. Kamra HT, Gadgil PA, Chaware SA, Kolhe AB, Sakinlawar PW. Cytological correlation of early changes in spontaneously infarcted fibroadenoma-a rare case report. Ecancer 2012;6:257.

7. Walke V, Ramraje S, Jadhao V, Gupta S. Spontaneous infarction in fibroadenoma, a diagnostic challenge on cytology: Report of two ca ses. J Health Spec 2014;2:8688. 